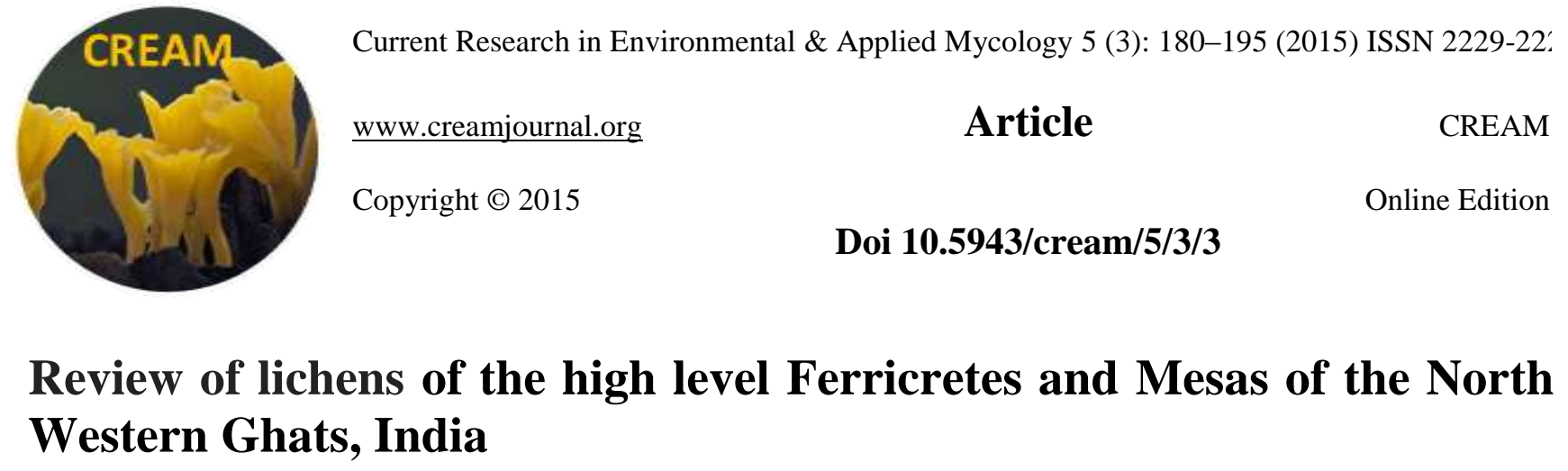

\title{
Pandit G
}

Biodiversity \& Palaeobiology Group, Agharkar Research Institute, G.G. Agarkar Road, Pune 411004, India.

Pandit G 2015 - Review of lichens of the high level Ferricretes and Mesas of the North Western Ghats, India. Current Research in Environmental \& Applied Mycology 5(3), 180-195, Doi $10.5943 / \mathrm{cream} / 5 / 3 / 3$

\begin{abstract}
The paper includes 234 species in 61 genera belonging to 30 families reported from the high level Ferricretes and basalt mesas, of the North Western Ghats of Maharashtra. The average percentage of the lichen species in Maharashtra on high level Ferricretes is $22.91 \%$ and on the basalt Mesas is $0.682 \%$. Of these 234 species, 50 species are new to science, reported from these plateau areas and 25 species have their type locality in and around the plateaus.
\end{abstract}

Key words - basalt - laterite - lichenized fungi - rocky outcrops.

\section{Introduction}

The Western Ghats of India are one of the important biodiversity hot spots of the world and comprise many rocky plateaus. They are a highly specialized terrestrial island-like habitat, with a unique combination of microhabitats that support endemic biodiversity due to the geology, geomorphology, climate and edaphic conditions. The flora and fauna has diversified in to narrowniched endemics in this habitat. Cycling of nutrients and of water in this habitat is also different than any other habitats, such as forest or grasslands. Scientific recognition of this uniqueness has come only within the last decade (Watve 2013). They are categorized and treated as wastelands, as they remain dry and look barren for most part of the year.

In the northern part of the Western Ghats and in the Konkan region of Maharashtra State, rocky plateaus are a very prominent part of the landscape. Ferricretes, often known as "tablelands" owing to the wide flat appearance and steep edges are common. There are a total of 67 plateau sites or rocky outcrops in the North Western Ghats and Konkan region of Maharashtra.

These plateaus are of the following three types:

High-level Ferricretes (HLF) occurs on high-level Laterites between $15-18^{\circ} 20^{\prime} \mathrm{N}$, extend inland to $74^{\circ} \mathrm{E}$, and are located between 800 and $1400 \mathrm{~m}$ (Widdowson \& Cox 1996) in the districts of Satara, Kolhapur, Sangli, Ratnagiri, and Sindhudurg, which include the crestline of the NW Ghats. Of these 26 plateaus are high level Ferricretes. The high level Ferricretes that are explored are Amba, Amboli, Kas, Mahabaleshwar-Panchgani and Panhala (Table 1, Fig. 1). 
Table 1 High level Ferricretes

\begin{tabular}{|c|c|c|c|c|c|c|}
\hline Plateaus & & $\begin{array}{c}\text { Microlichen } \\
\text { taxa }\end{array}$ & $\begin{array}{c}\text { Macrolichen } \\
\text { taxa }\end{array}$ & $\begin{array}{c}\text { Total number of } \\
\text { lichen taxa }\end{array}$ & $\begin{array}{c}\% \text { in } \\
\text { Maharashtra }\end{array}$ & $\begin{array}{l}\% \text { in } \\
\text { India }\end{array}$ \\
\hline Panhala & & 30 & 33 & 63 & 21.87 & 2.73 \\
\hline Amboli & & 64 & 24 & 88 & 30.55 & 3.82 \\
\hline Amba & & 24 & 6 & 30 & 10.41 & 1.30 \\
\hline Kas & & 5 & 14 & 19 & 6.59 & 0.82 \\
\hline $\begin{array}{l}\text { Mahabaleshwar } \\
\text { Panchgani }\end{array}$ & $\&$ & 72 & 57 & 130 & 45.13 & 5.64 \\
\hline
\end{tabular}

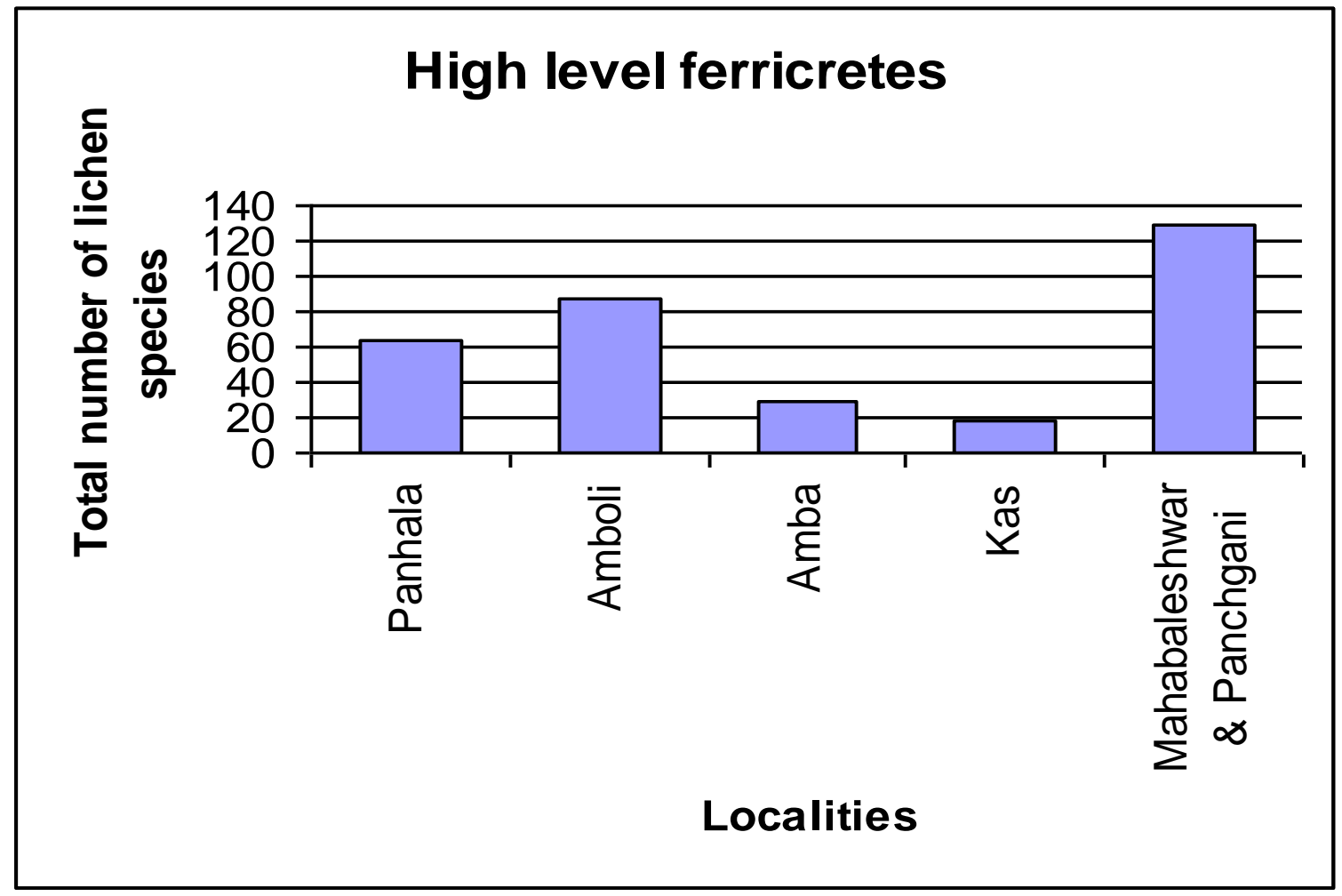

Fig. 1 - Graphical representation of high level Ferricretes

Basalt Mesa (BM) outcrops are exposed on mesas between $18^{\circ} 20^{\prime}-21^{\circ} \mathrm{N} \& 73^{\circ} 35^{\prime}-73^{\circ} 50^{\prime} \mathrm{E}$. These are areas where the upper layers of rocks have eroded to expose the underlying basalt flows, and hence can occur at any altitude depending upon the degree of weathering. The basalt mesas that are explored are Anjaneri, Matheran, Durgawadi and Malshej ghat. There are 10 basalt Mesas and the remaining three are between high level Ferricretes to low level Ferricretes (Table 2, Fig. 2).

Table 2 Basalt Mesas

\begin{tabular}{lccccc}
\hline \multicolumn{1}{c}{ Plateaus } & Microlichen taxa & Macrolichen taxa & $\begin{array}{c}\text { Total number of } \\
\text { lichen taxa }\end{array}$ & $\begin{array}{c}\text { \% in Maharashtra } \\
\text { \% in } \\
\text { India }\end{array}$ \\
\hline Anjaneri & 6 & 6 & 12 & 4.16 & 0.52 \\
Durgawadi & 10 & 14 & 24 & 8.33 & 1.04 \\
Malshej ghat & 11 & 10 & 21 & 7.29 & 0.911 \\
Matheran & 3 & 3 & 6 & 2.08 & 0.26 \\
\hline
\end{tabular}




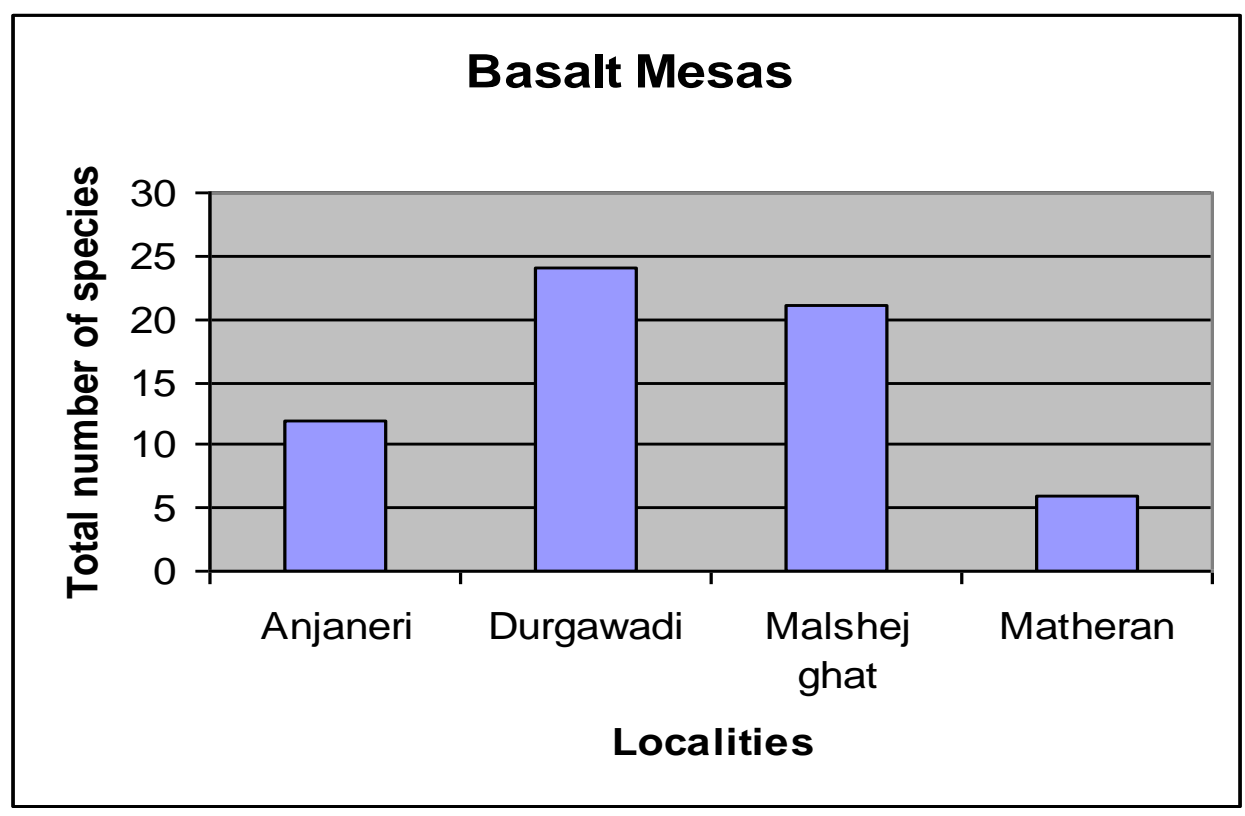

Fig. 2 - Graphical representation of basalt Mesas

Low-level Ferricretes (LLF) occur in the low-level laterites of Konkan plains between 50-200 m south of $18^{\circ} 20^{\prime} \mathrm{N}$ in the Raigad, Ratnagiri and Sindhudurg districts, as well as all of Karnataka and the Kerala coast, extending from the sea coast to the foothills of the Western Ghats and 28 plateaus are low level Ferricretes in Maharashtra.

Many of the hill forts of Maharashtra State have exposed hilltops of basalt (Watve 2013).

Lichens are regarded as an example of controlled parasitism (where in a fungal partner, the mycobiont and one or more photosynthetic partners, the photobiont, are present to form a complex structure forming- "symbiont". They play an important role in the mineral cycling patterns of their ecosystem. More than 2300 lichen species are presently known from India (Singh \& Sinha 2010) and a total of 288 lichen species in 68 genera are known from the State of Maharashtra (Makhija et al. 2014).

The plateaus have a unique lichen flora, and are also facing threats from tourism, mining, and grazing (Table 3). Many organizations and researchers working on these plateau sites are actively helping in conserve them. The Maharashtra forest department has also taken a leading role in the region by identifying rocky plateaus of special conservation significance and taking steps to include details of rocky plateaus in the regional working plans.

Table 3 Details of Explored plateaus

\begin{tabular}{|c|c|c|c|c|c|c|c|}
\hline Regions & Villages & $\begin{array}{l}\text { Local } \\
\text { names }\end{array}$ & District & $\begin{array}{l}\text { Outcrop } \\
\text { type }\end{array}$ & $\begin{array}{l}\text { Land } \\
\text { ownership }\end{array}$ & Threat & $\begin{array}{l}\text { Disturbanc } \\
\text { e }\end{array}$ \\
\hline $\begin{array}{l}\text { Nashik } \\
\text { area }\end{array}$ & Anjaneri & Anjaneri & Nashik & $\mathrm{BM}$ & Partly RF & Tourism & Low \\
\hline Kas area & $\begin{array}{l}\text { Kas (Site } \\
\mathrm{K})\end{array}$ & $\begin{array}{l}\text { Apti papdi, } \\
\text { dhang sada, } \\
\text { thanoba sada }\end{array}$ & Satara & HLF & RF, private & Flower tourism & Moderate \\
\hline $\begin{array}{l}\text { Panchgani } \\
\text { area }\end{array}$ & $\begin{array}{l}\text { Godawali } \\
\text { / } \\
\text { Panchgan } \\
\text { i }\end{array}$ & $\begin{array}{l}\text { Panchgani } \\
\text { Tableland/ } \\
\text { Asia plateau }\end{array}$ & Satara & HLF & $\begin{array}{l}\text { Mahabaleshwa } \\
\text { r Panchgani } \\
\text { ecosensitive } \\
\text { zone } \\
\text { Declared } \\
\text { natural } \\
\text { heritage, } \\
\text { Conservation }\end{array}$ & $\begin{array}{l}\text { Commercial } \\
\text { landscape } \\
\text { tourism }\end{array}$ & Very high \\
\hline
\end{tabular}




\begin{tabular}{|c|c|c|c|c|c|c|c|}
\hline Regions & Villages & $\begin{array}{l}\text { Local } \\
\text { names }\end{array}$ & District & $\begin{array}{l}\text { Outcrop } \\
\text { type }\end{array}$ & $\begin{array}{l}\text { Land } \\
\text { ownership }\end{array}$ & Threat & $\begin{array}{l}\text { Disturbanc } \\
\text { e }\end{array}$ \\
\hline $\begin{array}{l}\text { Malshej } \\
\text { Ghat area }\end{array}$ & Malshej & $\begin{array}{l}\text { Malshej } \\
\text { Ghat }\end{array}$ & $\begin{array}{l}\text { Ahemdnaga } \\
\mathrm{r}\end{array}$ & $\mathrm{BM}$ & $\begin{array}{l}\text { zone } \\
\text { Mostly private }\end{array}$ & Tourism & High \\
\hline Panhala & Fort & Fort & Kolhapur & HLF & Reserve Forest & $\begin{array}{l}\text { Grazing by } \\
\text { cattle/Township } \\
\text { / ESZ1 }\end{array}$ & Moderate \\
\hline $\begin{array}{l}\text { Amba } \\
\text { ghat area }\end{array}$ & Amba & - & Kolhapur & $\begin{array}{l}\text { Secondar } \\
\text { y } \\
\text { laterite }\end{array}$ & Reserve Forest & Tourism & Moderate \\
\hline $\begin{array}{l}\text { Durgawad } \\
\text { i }\end{array}$ & $\begin{array}{l}\text { Ambe- } \\
\text { Hatviji/ } \\
\text { Junnar }\end{array}$ & Killa /Fort & Pune & $\mathrm{BM}$ & $\begin{array}{l}\text { Private and } \\
\text { Cultivated for } \\
\text { rice }\end{array}$ & $\begin{array}{l}\text { Grazing, Fires, } \\
\text { Blasting for } \\
\text { ponds }\end{array}$ & $\begin{array}{l}\text { Local } \\
\text { grazing, } \\
\text { fires } \\
\text { trampling by } \\
\text { tourists, } \\
\text { plant } \\
\text { collection } \\
\text { and } \\
\text { proposed } \\
\text { wind farms }\end{array}$ \\
\hline Amboli & Amboli & $\begin{array}{l}\text { Choukul } \\
\text { sada, } \\
\text { Hiranyakesh } \\
\text { i sada, } \\
\text { Khamtyahca } \\
\text { sada }\end{array}$ & Sindhudurg & HLF & Reserve Forest & Grazing & Low \\
\hline $\begin{array}{l}\text { Raigad } \\
\text { District }\end{array}$ & Matheran & Matheran & Raigad & $\begin{array}{l}\text { Between } \\
\text { HLF and } \\
\text { LLF }\end{array}$ & $\begin{array}{l}\text { Matheran Eco } \\
\text { Sensitive Zone }\end{array}$ & Tourism & High \\
\hline
\end{tabular}

\section{Review of Literature}

The Maharashtra State was investigated for lichens during 2001-2008, and published as "Lichens of Maharashtra" (Makhija et al. 2014). Few plateaus were also explored but were of a floristic nature and plateaus or rocky outcrops were not taken into account as specialized habitats. Hence, lichen data was not exclusively available for the plateaus. Recently a list on lichens of the Mahabaleshwar-Panchgani Ecosensitive zone was published (Pandit 2014b), also two new generic records have been reported for the two high level Ferricretes (Pandit 2014a). However, the low level Ferricretes have not been surveyed in the concept of specialized habitats and collections have been made in and around the plateau and not on the actual plateau.

Explorations of the rocky plateaus have so far concentrated on angiosperms, amphibians and reptiles (Bhattaria et al. 2012, Giri et al. 2004, 2008, Gower et al. 2007, Lekhak \& Yadav 2012, Yadav \& Sardesai 2002). New species of vertebrates, invertebrates, angiosperms and pteridophytes have been reported from rocky plateaus in the study area, indicating their biodiversity richness. However, the cryptogamic vegetation not been studied in detail. A cryptogamic crust covers a large portion of rocky plateaus and it is considered as one of the major communities on rock outcrops world over (Porembski et al. 2000).

Büdel has remarked on the surprisingly high variety of cyanobacteria and cyano- bacterial lichens on outcrops across the tropical regions. The lichen diversity of rock outcrops has never specifically been reviewed. In view of this, the paper presents a review of lichens reported on rocky plateau sites. It will serve as a baseline for future studies on this subject. By using the outcrop data of Watve (2013) as the baseline data, this review produces a list of lichens from these poorly investigated plateaus.

\section{Materials \& Methods}

For the preparation of this review paper, primary, as well as secondary data has been used. During the surveys conducted for the compilation of book "Lichens of Maharashtra" vouchers 
Table 4 List of lichens occurring on various substrates

\begin{tabular}{|c|c|c|c|c|}
\hline Taxa & Saxicolous & Corticolous & Terricolous & Muscicolous \\
\hline Aspicilia calcarea & + & & & \\
\hline Caloplaca abuensis & + & & & \\
\hline Caloplaca amarkantaka & + & & & \\
\hline Caloplaca cupulifera & + & & & \\
\hline Cladonia sp. & & & + & \\
\hline Cladonia scabriuscula & + & & + & \\
\hline Collema furfureolum & + & & & \\
\hline Collema polycarpon & + & & & \\
\hline Collema tenax var. tenax & + & & & \\
\hline Collema texanum var. texanum & + & & & \\
\hline Diploschistes rampodensis & + & & & \\
\hline Diploschistes cf. rampodensis & + & & & \\
\hline Immersaria cf olivacea & + & & & \\
\hline Koerberiella wimmeriana & + & & & \\
\hline Lepraria lobificans & + & & & \\
\hline Leptogium burnetiae var. hirsutum & & & & + \\
\hline Leptogium chloromelum & & & & + \\
\hline Leptogium cyanescens & & + & & + \\
\hline Leptogium denticulatum & & + & & + \\
\hline Leptogium patwardhanii & & & & + \\
\hline Leptogium ulvaceum & & & + & \\
\hline Leptogium verrucosum & + & + & & \\
\hline Parmotrema tinctorum & + & + & & \\
\hline Pertusaria corallina & + & & & \\
\hline Porina sp. & + & & & \\
\hline Ramalina sp. & & & + & \\
\hline Staurothele clopima & + & & & \\
\hline Staurothele fissa & + & & & \\
\hline Thelenella sp. & + & & & \\
\hline Trapelia placodioides & + & & & \\
\hline Verrucaria acrotella & + & & & \\
\hline
\end{tabular}

specimens were collected and deposited at Ajrekar Mycological herbarium (AMH) lodged at ARI. These primary occurrence records together with field ecological data on lichen habitats, collected during various survey tours from 2001 to 2014 has been analyzed here. Currently work is going on as part of the funded project from the Department of Science \& Technology, Govt. of India, project on the two outcrops of Kas and Panchgani, where lichen explorations and ecological data collections were made. In addition to this, regional research on lichens since 2000-2008 has been reviewed for this compilation.

\section{Results and Discussion}

The secondary data sources on lichens are voluminous. However, they deal mainly with taxonomy and nomenclature issues. There is a dearth of ecological information beyond lichens forms and substrates. Only 9-10 rocky plateaus have been repeatedly surveyed by lichenologists. Still a very large number of 234 species in 61 genera belonging to 30 families are reported from the nine plateau regions of NW Ghats of Maharashtra. This clearly indicates the importance of this habitat for lichen biodiversity and need for further detailed studies systematically from this habitat.

The dominant families for the few studied plateau regions are Graphidaceae with highest species number of 47 species, followed by Physciaeae- 31 species, Collemataceae with 23 species, Lecanoraceae 18 species, Parmeliaceae with 15 species, Thelotremoid-Graphidaceae with 14 species and Ramalinaceae with 13 species. The average percentage of species on high level Ferricretes is $22.91 \%$ and on the basalt Mesas is $0.68 \%$, whereas the low level Ferricretes plateaus in Maharashtra mentioned by Watve (2013) have not been well-studied, but their surroundings are explored for lichens so we do not have lichen reports from specific plateaus as such. 
Table 5 Comparative lichen data of the high level Ferricretes and basalt Mesas [1 Amba, 2 Amboli, 3 Kas, 4 Mahabaleshwar-Panchgani, 5 Panhala, 6 Anjaneri, 7 Matheran, 8 Durgawadi, 9 Malshej ghat]

\begin{tabular}{|c|c|c|c|c|c|c|c|c|c|c|c|c|c|c|}
\hline \multirow[t]{2}{*}{ Families } & \multirow[t]{2}{*}{ Genera } & \multirow[t]{2}{*}{ Species } & \multirow[t]{2}{*}{ Names of Taxon } & \multirow[t]{2}{*}{ Growth forms } & \multicolumn{5}{|c|}{ HLF } & \multicolumn{4}{|c|}{ BM } & \multirow[t]{2}{*}{ Status } \\
\hline & & & & & 1 & 2 & 3 & 4 & 5 & 6 & 7 & 8 & 9 & \\
\hline \multirow[t]{7}{*}{ Arthoniaceae } & 2 & 7 & Arthothelium albescens Patw. \& Makhija & Crustose & & & & + & & & & & & Restricted \\
\hline & & & Arthothelium awasthii Patw. \& Makhija & Crustose & & + & & & + & & & + & & \\
\hline & & & $\begin{array}{l}\text { Arthothelium deplanatum (Müll. Arg.) } \\
\text { Makhija \& Patw. }\end{array}$ & Crustose & & & & & + & + & & & & \\
\hline & & & $\begin{array}{l}\text { Arthothelium nigrodiscum Patw. \& } \\
\text { Makhija }\end{array}$ & Crustose & + & & & + & & & & & & \\
\hline & & & Arthothelium saxicola Makhija \& Patw. & Crustose & & & & & & & & + & & Restricted \\
\hline & & & $\begin{array}{l}\text { Arthothelium subruanum Makhija \& } \\
\text { Patw. }\end{array}$ & Crustose & & & & + & & & & & & Restricted \\
\hline & & & $\begin{array}{l}\text { Cryptothecia lunulata (Zahlbr.) Makhija } \\
\text { \& Patw. }\end{array}$ & Crustose & & & & + & & & & & & Restricted \\
\hline \multirow[t]{3}{*}{ Arthopyreniaceae } & 1 & 3 & $\begin{array}{l}\text { Mycomicrothelia exigua (Müll. Arg.) D. } \\
\text { Hawksw. }\end{array}$ & Crustose & + & + & + & & & & & & & \\
\hline & & & $\begin{array}{l}\text { Mycomicrothelia hemispherica (Mull. } \\
\text { Arg.) D. Hawksw. }\end{array}$ & Crustose & & & & + & & & & & & Restricted \\
\hline & & & $\begin{array}{l}\text { Mycomicrothelia obovata (Stirt.) D. } \\
\text { Hawksw. }\end{array}$ & Crustose & & + & & & & & & & & Restricted \\
\hline Brigantiaceae & 1 & 1 & $\begin{array}{l}\text { Brigantiaea patwardhanii Chitale \& } \\
\text { Makhija }\end{array}$ & Crustose & & + & & & & & & & & Restricted \\
\hline \multirow[t]{3}{*}{ Caliciaceae } & 1 & 3 & Buellia panchganiensis Makhija \& Dube & Crustose & & & & + & & & & & & Restricted \\
\hline & & & Buellia sp. B & Crustose & & & & & + & & & & & Restricted \\
\hline & & & Buellia tabularis Makhija \& Dube & Crustose & & & & + & & & & & & Restricted \\
\hline \multirow[t]{2}{*}{ Cladoniaceae } & 1 & 2 & Cladonia scabriuscula (Delise) Nyl. & Fruticose & & & & + & & & & & & Restricted \\
\hline & & & Cladonia sp. & Fruticose & & & & + & & & & & & Restricted \\
\hline \multirow[t]{2}{*}{ Coccocarpiaceae } & 1 & 2 & $\begin{array}{l}\text { Coccocarpia erythroxyli (Spreng.) } \\
\text { Swinscow \& Krog }\end{array}$ & Foliose & & + & & & & & & & & Restricted \\
\hline & & & $\begin{array}{l}\text { Coccocarpia palmicola (Spreng.) Arv. \& } \\
\text { D. J. Gall. }\end{array}$ & Foliose & & & & & + & & & & & Restricted \\
\hline \multirow[t]{4}{*}{ Collemataceae } & 2 & 25 & $\begin{array}{l}\text { Collema conglomeratum Hoffm. var. } \\
\text { crassiusculum (Malme) Degel. }\end{array}$ & Foliose & & & & + & & & & & & Restricted \\
\hline & & & Collema furfureolum Mull. Arg. & Foliose & & & & + & & & & & & Restricted \\
\hline & & & $\begin{array}{l}\text { Collema leptaleum Tuck. var. biliosum } \\
\text { (Mont.) Degel. }\end{array}$ & Foliose & & & & + & & & & & & Restricted \\
\hline & & & $\begin{array}{l}\text { Collema polycarpon Hoffm. var. } \\
\text { polycarpon }\end{array}$ & Foliose & & & & + & & & & & & Restricted \\
\hline
\end{tabular}




\begin{tabular}{|c|c|c|c|c|c|c|c|c|c|c|c|c|c|c|}
\hline \multirow[t]{2}{*}{ Families } & \multirow[t]{2}{*}{ Genera } & \multirow[t]{2}{*}{ Species } & \multirow[t]{2}{*}{ Names of Taxon } & \multirow[t]{2}{*}{ Growth forms } & \multicolumn{5}{|c|}{ HLF } & \multicolumn{4}{|c|}{ BM } & \multirow[t]{2}{*}{ Status } \\
\hline & & & & & 1 & 2 & 3 & 4 & 5 & 6 & 7 & 8 & 9 & \\
\hline & & & $\begin{array}{lccc}\text { Collema } & \text { pulcellum } & \text { Ach. } & \text { var. } \\
\text { subniarescens (Mull. Arg.) Degel. } & \end{array}$ & Foliose & & & & + & & & & & & Restricted \\
\hline & & & Collema tenax var. tenax (Sw.) Ach. & Foliose & & & & + & & & & & & Restricted \\
\hline & & & Collema texanum var. texanum Tuck. & Foliose & & & & + & & & & & & Restricted \\
\hline & & & $\begin{array}{l}\text { Leptogium austroamericanum (Malme) } \\
\text { Dodge }\end{array}$ & Foliose & & + & & & & & & & & Restricted \\
\hline & & & Leptogium azureum (Sw.) Mont. & Foliose & & + & & + & & & & & & \\
\hline & & & $\begin{array}{l}\text { Leptogium burnetiae var. hirsutum } \\
\text { (Sierk) P.M. Jørg. }\end{array}$ & Foliose & & + & & + & + & & & & + & Common \\
\hline & & & Leptogium chloromelum (Sw.) Nyl. & Foliose & & & & + & & & & & & Restricted \\
\hline & & & $\begin{array}{l}\text { Leptogium cochleatum (Dicks.) P.M. } \\
\text { Jorg. \& P. James }\end{array}$ & Foliose & & & & + & & & & & & Restricted \\
\hline & & & Leptogium cyanescens (Ach.) Korb. & Foliose & & + & & + & + & & & & & \\
\hline & & & Leptogium denticulatum Nyl. & Foliose & & & & + & & & & & + & \\
\hline & & & $\begin{array}{l}\text { Leptogium gelatinosum (With.) J.R. } \\
\text { Laudon }\end{array}$ & Foliose & & & & + & & & & & & Restricted \\
\hline & & & Leptogium indicum Awasthi \& Akthar & Foliose & & & + & + & & & & & & \\
\hline & & & Leptogium javanicum Mont & Foliose & & & + & + & + & + & & & + & Common \\
\hline & & & $\begin{array}{l}\text { Leptogium patwardhanii A. Dube \& } \\
\text { Makhija }\end{array}$ & Foliose & & + & & & & & & & & Restricted \\
\hline & & & Leptogium phyllocarpum (Pers.) Mont. & Foliose & & & & + & & & & & & Restricted \\
\hline & & & Leptogium propaguliferum Vain. & Foliose & & + & & + & + & + & & & + & Common \\
\hline & & & $\begin{array}{l}\text { Leptogium subazureum A. Dube \& } \\
\text { Makhija }\end{array}$ & Foliose & + & + & & + & + & & & & + & Common \\
\hline & & & Leptogium ulvaceum (Pers.) Vain. & Foliose & & & & + & & & & & & Restricted \\
\hline & & & Leptogium verrucosum A. Dube \& Makhija & Foliose & & & & & & & & + & & Restricted \\
\hline \multirow[t]{7}{*}{ Graphidaceae } & 8 & 47 & $\begin{array}{l}\text { Carbacanthographis awasthii (Patw. \& } \\
\text { Nagarkar) Chitale \& Makhija }\end{array}$ & Crustose & & + & & & & & & & & Restricted \\
\hline & & & Diorygma "microsporum" ad int. & Crustose & & & & + & & & & & & Restricted \\
\hline & & & Diorygma "patwardhanii" ad int & Crustose & & & & + & & & & & & Restricted \\
\hline & & & $\begin{array}{l}\text { Diorygma albocinerascens Makhija, } \\
\text { Chitale \& B.O. Sharma }\end{array}$ & Crustose & & + & & + & + & & & + & + & Common \\
\hline & & & $\begin{array}{l}\text { Diorygma albovirescens Makhija, Chitale } \\
\text { \& B.O. Sharma }\end{array}$ & Crustose & & & & + & & & & & & Restricted \\
\hline & & & $\begin{array}{l}\text { Diorygma excipuloconvergentum } \\
\text { Makhija, Chitale \& B.O. Sharma }\end{array}$ & Crustose & + & + & & + & + & & & + & + & Common \\
\hline & & & $\begin{array}{l}\text { Diorygma junghuhnii (Mont. \& Bosch) } \\
\text { Kalb. in Kalb }\end{array}$ & Crustose & & & & + & & & & & & Restricted \\
\hline
\end{tabular}




\begin{tabular}{|c|c|c|c|c|c|c|c|c|c|c|c|c|c|c|}
\hline \multirow[t]{2}{*}{ Families } & \multirow[t]{2}{*}{ Genera } & \multirow[t]{2}{*}{ Species } & \multirow[t]{2}{*}{ Names of Taxon } & \multirow[t]{2}{*}{ Growth forms } & \multicolumn{5}{|c|}{ HLF } & \multicolumn{4}{|c|}{ BM } & \multirow[t]{2}{*}{ Status } \\
\hline & & & & & 1 & 2 & 3 & 4 & 5 & 6 & 7 & 8 & 9 & \\
\hline & & & $\begin{array}{l}\text { Diorygma megaspermum } \quad \text { Makhija, } \\
\text { Chitale \& B.O. Sharma }\end{array}$ & Crustose & & + & & + & & & & & & \\
\hline & & & $\begin{array}{l}\text { Diorygma megasporum Kalb, Staiger \& } \\
\text { Elix }\end{array}$ & Crustose & + & + & & + & + & & & & & Common \\
\hline & & & $\begin{array}{l}\text { Diorygma megistosporum Makhija, } \\
\text { Chitale \& B.O. Sharma }\end{array}$ & Crustose & & & & + & + & & & & & \\
\hline & & & $\begin{array}{l}\text { Diorygma panchganiense Makhija, } \\
\text { Chitale \& B.O. Sharma }\end{array}$ & Crustose & & & & + & & & & & & Restricted \\
\hline & & & $\begin{array}{l}\text { Diorygma rufosporum (Patw. \& C.R. } \\
\text { Kulk.) B.O. Sharma \& Makhija }\end{array}$ & Crustose & & + & & & & & & & & Restricted \\
\hline & & & Fissurina cingalina (Nyl.) Staiger & Crustose & & + & & + & & & & & & \\
\hline & & & Glyphis cicatricosa Ach. & Crustose & & + & & & & & & & & Restricted \\
\hline & & & Graphis ajarekarii Patw. \& C.R. Kulk. & Crustose & & + & & & & & & & & Restricted \\
\hline & & & Graphis aurita Eschw. in Martius & Crustose & & + & & & & & & & & Restricted \\
\hline & & & Graphis duplicata Ach. & Crustose & & & & + & & & & & & Restricted \\
\hline & & & Graphis elevativerrucosa Chitale \& al & Crustose & & + & & & & & & & & Restricted \\
\hline & & & Graphis galactoderma (Zahlbr.) Lucking & Crustose & & & & & + & & & & & Restricted \\
\hline & & & Graphis lineola Ach. & Crustose & & & & + & & & & & & Restricted \\
\hline & & & Graphis maharashtrana Chitale \& al. & Crustose & & & & & + & + & & & + & \\
\hline & & & Graphis nigroglauca Leight. & Crustose & & & & + & & & & & & Restricted \\
\hline & & & $\begin{array}{l}\text { Graphis panhalensis (Patw. \& C.R. } \\
\text { Kulk.) Chitale \& al. }\end{array}$ & Crustose & & & & & + & & & & & Restricted \\
\hline & & & Graphis parilis Kremph. & Crustose & & & & + & & & & & & Restricted \\
\hline & & & Graphis platycarpa Eschw. & Crustose & & & & + & & & & & & Restricted \\
\hline & & & $\begin{array}{l}\text { Graphis polystriata Makhija, A. Dube, } \\
\text { Adaw. \& Chitale }\end{array}$ & Crustose & + & + & + & + & + & & + & & + & Common \\
\hline & & & Graphis proserpens Vain. & Crustose & & & & + & & & & & & Restricted \\
\hline & & & Graphis sp. 1 & Crustose & & & & + & & & & & & Restricted \\
\hline & & & Graphis subducta Vain. & Crustose & & & & & + & & & & & Restricted \\
\hline & & & Graphis subserpentina (Nyl.) Mull Arg. & Crustose & & & & + & & & & & & Restricted \\
\hline & & & Graphis treblocarpa (Bel.) Nyl. & Crustose & & & & + & & & & & & Restricted \\
\hline & & & Graphis tsunodae Zahlbr. & Crustose & & & & + & & & & & & Restricted \\
\hline & & & Graphis vittata Vain. & Crustose & & & & & + & & & & & Restricted \\
\hline & & & $\begin{array}{l}\text { Hemithecium amboliense Makhija \& A. } \\
\text { Dube }\end{array}$ & Crustose & & + & & & & & & & & Restricted \\
\hline & & & $\begin{array}{l}\text { Hemithecium aphanes (Mont. et Bosch) } \\
\text { M. Nakan. \& Kashiw. }\end{array}$ & Crustose & + & + & & & & & & & & \\
\hline & & & $\begin{array}{l}\text { Hemithecium epixanthum (Mont. \& } \\
\text { Bosch) Chitale \& Makhija }\end{array}$ & Crustose & & + & & & + & & & & + & \\
\hline
\end{tabular}




\begin{tabular}{|c|c|c|c|c|c|c|c|c|c|c|c|c|c|c|}
\hline \multirow[t]{2}{*}{ Families } & \multirow[t]{2}{*}{ Genera } & \multirow[t]{2}{*}{ Species } & \multirow[t]{2}{*}{ Names of Taxon } & \multirow[t]{2}{*}{ Growth forms } & \multicolumn{5}{|c|}{ HLF } & \multicolumn{4}{|c|}{ BM } & \multirow[t]{2}{*}{ Status } \\
\hline & & & & & 1 & 2 & 3 & 4 & 5 & 6 & 7 & 8 & 9 & \\
\hline & & & $\begin{array}{l}\text { Hemithecium microspermum Chitale, } \\
\text { Makhija \& B.O. Sharma }\end{array}$ & Crustose & & + & & & & & & & & Restricted \\
\hline & & & $\begin{array}{l}\text { Hemithecium multistriatum (Müll. Arg.) } \\
\text { Chitale \& Makhija }\end{array}$ & Crustose & & + & & & & & & & & Restricted \\
\hline & & & $\begin{array}{l}\text { Hemithecium nakanishianum (Patw. \& } \\
\text { C.R. Kulk.) Makhija \& A. Dube }\end{array}$ & Crustose & + & + & & + & + & & + & & & Common \\
\hline & & & $\begin{array}{l}\text { Hemithecium norsticticum Makhija \& A. } \\
\text { Dube }\end{array}$ & Crustose & + & & & + & & & & & + & \\
\hline & & & $\begin{array}{l}\text { Hemithecium pyrrhochroa (Mont. \& } \\
\text { Bosch.) V. Tewari \& Upreti }\end{array}$ & Crustose & & & & + & & & & & & Restricted \\
\hline & & & $\begin{array}{l}\text { Hemithecium salacinilabiatum (Patw. \& } \\
\text { C.R. Kulk.) Chitale \& Makhija }\end{array}$ & Crustose & & + & & & & & & & & Restricted \\
\hline & & & $\begin{array}{l}\text { Hemithecium stictilabiatum (Patw. \& } \\
\text { C.R. Kulk.) Chitale \& Makhija }\end{array}$ & Crustose & + & + & & & & & & & & \\
\hline & & & $\begin{array}{l}\text { Pallidogramme commutabilis (Kremp.) } \\
\text { Chitale \& Makhija }\end{array}$ & Crustose & & & & + & & & & & & Restricted \\
\hline & & & $\begin{array}{l}\text { Pallidogramme indica A. Dube \& } \\
\text { Makhija }\end{array}$ & Crustose & + & + & & & & & & & & \\
\hline & & & $\begin{array}{l}\text { Pallidogramme undulatolirellatum A. } \\
\text { Dube \& Makhija }\end{array}$ & Crustose & + & + & & & & & & & & \\
\hline & & & $\begin{array}{l}\text { Platygramme halei (Patw. \& C.R. Kulk.) } \\
\text { Makhija \& Chitale }\end{array}$ & Crustose & & + & & & & & & & & Restricted \\
\hline \multirow[t]{15}{*}{ Lecanoraceae } & 1 & 18 & Lecanora alba Lumbsch & Crustose & & & & + & & & & & & Restricted \\
\hline & & & Lecanora allophana (Ach.) Röhl. & Crustose & & & & + & & & & & & Restricted \\
\hline & & & Lecanora andina Rasanen & Crustose & & & & + & & & & & & Restricted \\
\hline & & & $\begin{array}{l}\text { Lecanora austrointumescens Lumbsch \& } \\
\text { Elix }\end{array}$ & Crustose & & & & + & & & & & & Restricted \\
\hline & & & Lecanora cenisia Ach. & Crustose & & & + & + & & & & + & & \\
\hline & & & Lecanora cf. chlarotera Nyl. & Crustose & & & & + & & & & & & Restricted \\
\hline & & & Lecanora cf. fimbriatula Stirt. & Crustose & + & + & + & & + & & + & + & & Common \\
\hline & & & Lecanora cf. imshaugii Brodo & Crustose & & & + & + & + & + & & & + & Common \\
\hline & & & Lecanora cf. perplexa Brodo & Crustose & & & & & + & & & & & Restricted \\
\hline & & & Lecanora cf. xylophila Hue & Crustose & & & & & & & & & + & Restricted \\
\hline & & & Lecanora chlarotera Nyl. & Crustose & & & & + & + & & & & & \\
\hline & & & Lecanora expallens Ach. & Crustose & & & & + & & & & & & Restricted \\
\hline & & & Lecanora interjecta Mull. Arg. & Crustose & & & & + & & & & & & Restricted \\
\hline & & & Lecanora lavidofusca Mull. Arg. & Crustose & & & & + & & & & & & Restricted \\
\hline & & & Lecanora sp. 1 (Table Land) & Crustose & & & & + & & & & & & Restricted \\
\hline
\end{tabular}




\begin{tabular}{|c|c|c|c|c|c|c|c|c|c|c|c|c|c|c|}
\hline \multirow[t]{2}{*}{ Families } & \multirow[t]{2}{*}{ Genera } & \multirow[t]{2}{*}{ Species } & \multirow[t]{2}{*}{ Names of Taxon } & \multirow[t]{2}{*}{ Growth forms } & \multicolumn{2}{|c|}{ HLF } & \multicolumn{7}{|c|}{$\mathbf{B M}$} & \multirow[t]{2}{*}{ Status } \\
\hline & & & & & 1 & 2 & 3 & 4 & 5 & 6 & 7 & 8 & 9 & \\
\hline & & & Lecanora sp. A & Crustose & + & & & & & & & & & Restricted \\
\hline & & & Lecanora sp. B & Crustose & & + & & & + & + & & + & & Common \\
\hline & & & Lecanora xylophila Hue & Crustose & & + & & & & & & & & Restricted \\
\hline Megasporaceae & 1 & 1 & Aspicilia calcarea (L.) Sommerf. & Crustose & & & & + & & & & & & Restricted \\
\hline Monoblastiaceae & 1 & 1 & $\begin{array}{l}\text { Anisomeridium albisedum (Nyl.) R. C. } \\
\text { Harris }\end{array}$ & Crustose & & & & + & & & & & & Restricted \\
\hline \multirow[t]{3}{*}{ Pannariaceae } & 1 & 3 & $\begin{array}{l}\text { Parmeliella brisbanensis (Knight.) P.M. } \\
\text { Jorg. \& D.J. Galloway }\end{array}$ & $\begin{array}{l}\text { Crustose- } \\
\text { squamulose-foliose }\end{array}$ & & & & + & & & & & & Restricted \\
\hline & & & Parmeliella fuscata P. M. Jørg. & Squamulose & & + & & & & & & & & Restricted \\
\hline & & & $\begin{array}{l}\text { Parmeliella subfuscata A. Dube \& } \\
\text { Makhija }\end{array}$ & Squamulose & & + & & & & & & & & Restricted \\
\hline \multirow[t]{15}{*}{ Parmeliaceae } & 7 & 15 & Bulbothrix isidiza (Nyl.) Hale & Foliose & & + & & & + & & & & & \\
\hline & & & Bulbothrix meizospora (Nyl.) Hale & Foliose & & & & & + & & & & & Restricted \\
\hline & & & $\begin{array}{l}\text { Bulbothrix tabacina (Mont. \& Bosch) } \\
\text { Hale }\end{array}$ & Foliose & & & & & + & & & & & Restricted \\
\hline & & & $\begin{array}{l}\text { Myelochroa aurulenta (Tuck.) Elix \& } \\
\text { Hale }\end{array}$ & Foliose & & & & + & & & & & & Restricted \\
\hline & & & $\begin{array}{l}\text { Parmelinella simplicior (Hale) Elix \& } \\
\text { Hale }\end{array}$ & Foliose & + & + & & + & + & + & & + & + & Common \\
\hline & & & $\begin{array}{l}\text { Parmelinella wallichiana (Tayl.) Elix \& } \\
\text { Hale }\end{array}$ & Foliose & & & + & + & & & & & & \\
\hline & & & $\begin{array}{l}\text { Parmotrema kamatii Patw. \& A. V. } \\
\text { Prabhu }\end{array}$ & Foliose & & + & & & + & & & + & & \\
\hline & & & Parmotrema praesorediosum (Nyl.) Hale & Foliose & & & & & + & & & & & Restricted \\
\hline & & & Parmotrema reticulatum (Taylor) Choisy & Foliose & + & & & & & & & & & Restricted \\
\hline & & & Parmotrema sancti-angelii (Lynge) Hale & Foliose & & & & + & + & & & & & \\
\hline & & & Parmotrema tinctorum (Nyl.) Hale & Foliose & & & + & + & + & & & + & & Common \\
\hline & & & $\begin{array}{l}\text { Remototrachyna awasthi (Hale \& Patw.) } \\
\text { Divakar \& Crespo }\end{array}$ & Foliose & & & + & + & + & & + & & & Common \\
\hline & & & $\begin{array}{l}\text { Rimelia reticulata (Taylor) Hale \& A. } \\
\text { Fletcher }\end{array}$ & Foliose & & & & + & & & & & & Restricted \\
\hline & & & Usnea complanata (Mull. Arg.) Motyka & Foliose & & & + & + & & & & + & & \\
\hline & & & Usnea ghattensis G. Awasthi & Foliose & & & + & + & & & + & & & \\
\hline \multirow[t]{5}{*}{ Pertusariaceae } & 1 & 8 & Pertusaria alutacea (Kremph.) Zahlbr. & Crustose & & & & + & & & & & & Restricted \\
\hline & & & $\begin{array}{l}\text { Pertusaria cf. depressa (Fee) Mont. et } \\
\text { Bosch }\end{array}$ & Crustose & & & & + & & & & & & Restricted \\
\hline & & & Pertusaria cf. quassiae (Fée) Nyl. & Crustose & & & & & + & + & & & & \\
\hline & & & Pertusaria cinchonae Müll. Arg. & Crustose & & & & & & & & & + & Restricted \\
\hline & & & Pertusaria corallina (L.) Arnold & Crustose & & & & + & & & & & & Restricted \\
\hline
\end{tabular}




\begin{tabular}{|c|c|c|c|c|c|c|c|c|c|c|c|c|c|c|}
\hline \multirow[t]{2}{*}{ Families } & \multirow[t]{2}{*}{ Genera } & \multirow[t]{2}{*}{ Species } & \multirow[t]{2}{*}{ Names of Taxon } & \multirow[t]{2}{*}{ Growth forms } & \multicolumn{5}{|c|}{ HLF } & \multicolumn{4}{|c|}{ BM } & \multirow[t]{2}{*}{ Status } \\
\hline & & & & & 1 & 2 & 3 & 4 & 5 & 6 & 7 & 8 & 9 & \\
\hline & & & Pertusaria pertusa (L.) Tuck. & Crustose & & & & + & & & & & & Restricted \\
\hline & & & Pertusaria quassiae (Fée) Nyl. & Crustose & + & & & + & + & & & + & & Common \\
\hline & & & Pertusaria sp. C & Crustose & & + & & & & & & & & Restricted \\
\hline \multirow[t]{2}{*}{ Phlyctidaceae } & 1 & 2 & Phlyctis communis Chitale \& Makhija & Crustose & + & + & + & & + & & & & & Common \\
\hline & & & Phlyctis karnatakana S. Joshi \& Upreti & Crustose & & & & + & & & & & & Restricted \\
\hline \multirow[t]{21}{*}{ Physciaceae } & 7 & 32 & Dirinaria applanata (Fée) D. D. Awasthi & Foliose & & + & & & + & & & & & \\
\hline & & & $\begin{array}{l}\text { Heterodermia incana (Stirt.) D. D. } \\
\text { Awasthi }\end{array}$ & Foliose & & + & + & + & & & & + & & Common \\
\hline & & & $\begin{array}{l}\text { Heterodermia albicans (Pers.) Swinscow } \\
\text { \& Krog L. }\end{array}$ & Foliose & & & & + & & & & & & Restricted \\
\hline & & & $\begin{array}{l}\text { Heterodermia angustiloba (Miill. Arg.) } \\
\text { Awasthi }\end{array}$ & Foliose & & & & + & + & & & + & & \\
\hline & & & $\begin{array}{l}\text { Heterodermia antillarum (Vain.) } \\
\text { Swinscow \& Krog }\end{array}$ & Foliose & & & & & + & & & & & Restricted \\
\hline & & & $\begin{array}{l}\text { Heterodermia boryi (Fée) Kr. P. Singh \& } \\
\text { S. Singh }\end{array}$ & Foliose & & & + & + & & & & & & \\
\hline & & & $\begin{array}{l}\text { Heterodermia cf. japonica (M. Satô) } \\
\text { Swinscow \& Krog }\end{array}$ & Foliose & & & & + & & & & & & Restricted \\
\hline & & & $\begin{array}{ll}\text { Heterodermia diademata (Taylor) } \\
\text { Awasthi }\end{array}$ & Foliose & & + & + & + & + & + & & + & + & Common \\
\hline & & & $\begin{array}{l}\text { Heterodermia flabellata (Fée) D. D. } \\
\text { Awasthi }\end{array}$ & Foliose & & + & & & + & & & & & \\
\hline & & & $\begin{array}{l}\text { Heterodermia hypocaesia (Yesuda) D.D. } \\
\text { Awasthi }\end{array}$ & Foliose & & & & + & & & & & & Restricted \\
\hline & & & Heterodermia hypoleuca (Ach.) Trevis. & Foliose & & + & & & & & & & & Restricted \\
\hline & & & $\begin{array}{l}\text { Heterodermia japonica (Sato) Swinscow } \\
\text { \& Krog }\end{array}$ & Foliose & & & & & + & & & & & Restricted \\
\hline & & & Heterodermia leucomelos (L.) Poelt & Foliose & & & & + & & & & & & Restricted \\
\hline & & & Heterodermia obscurata (Nyl.) Trevis. & Foliose & + & & & & + & & & & & \\
\hline & & & Heterodermia podocarpa (Bel.) Awasthi & Foliose & & & + & + & & & + & + & & Common \\
\hline & & & $\begin{array}{l}\text { Heterodermia pseudospeciosa (Kurok.) } \\
\text { W. Culb. }\end{array}$ & Foliose & & + & + & + & + & & & + & & Common \\
\hline & & & Heterodermia sp. & Foliose & & & & + & & & & & & Restricted \\
\hline & & & Heterodermia speciosa (Wulfen) Trevis. & Foliose & & + & + & + & + & & & + & & Common \\
\hline & & & $\begin{array}{l}\text { Phaeophyscia endococcina var. } \\
\text { endococcinodes (Poelt) Moberg }\end{array}$ & Foliose & & & & + & & & & + & & \\
\hline & & & Phaeophyscia hispidula (Ach.) Moberg & Foliose & & + & & + & + & & & + & + & Common \\
\hline & & & $\begin{array}{l}\text { Phaeophyscia hispidula var. exornatula } \\
\text { (Zahlbr.) Moberg }\end{array}$ & Foliose & & & & & + & & & & & Restricted \\
\hline
\end{tabular}




\begin{tabular}{|c|c|c|c|c|c|c|c|c|c|c|c|c|c|c|}
\hline \multirow[t]{2}{*}{ Families } & \multirow[t]{2}{*}{ Genera } & \multirow[t]{2}{*}{ Species } & \multirow[t]{2}{*}{ Names of Taxon } & \multirow[t]{2}{*}{ Growth forms } & \multicolumn{5}{|c|}{ HLF } & \multicolumn{4}{|c|}{$\mathbf{B M}$} & \multirow[t]{2}{*}{ Status } \\
\hline & & & & & 1 & 2 & 3 & 4 & 5 & 6 & 7 & 8 & 9 & \\
\hline & & & $\begin{array}{l}\text { Phaeophyscia orbicularis (Neck.) } \\
\text { Moberg }\end{array}$ & Foliose & & & & & & & & & + & Restricted \\
\hline & & & $\begin{array}{l}\text { Phaeophyscia pyrrhophora (Poelt) D.D. } \\
\text { Awasthi \& M. Joshi }\end{array}$ & Foliose & & & & + & + & & & & & \\
\hline & & & Physcia abuensis D.D. Awasthi \& Singh & Foliose & & + & & + & + & & & & & \\
\hline & & & Physcia integrata Nyl. & Foliose & & & & + & + & + & & & & \\
\hline & & & Physcia tribacoides Nyl. & Foliose & & & & + & + & + & & + & + & Common \\
\hline & & & Physcia undulata Moberg & Foliose & & & & + & & & & & & Restricted \\
\hline & & & Physconia enteroxantha (Nyl.) Poelt & Foliose & & & & & + & & & & & Restricted \\
\hline & & & Pyxine cocoes var. cocoes (Swartz) Nyl. & Foliose & & & & + & & & & & & Restricted \\
\hline & & & $\begin{array}{l}\text { Pyxine cocoes var. prominula (Stirt.) D. } \\
\text { D. Awasthi }\end{array}$ & Foliose & & & & + & & & & & & Restricted \\
\hline & & & Pyxine petricola var. petricola Nyl. & Foliose & & & + & + & & & & & & \\
\hline Pilocarpaceae & 1 & 1 & Micarea sp. & Crustose & & & & + & & & & & & Restricted \\
\hline \multirow{9}{*}{ Porianaceae } & 1 & 9 & Porina africana Müll. Arg. & Crustose & + & + & & & & & & & & \\
\hline & & & Porina angusta Makhija \& al. & Crustose & & + & & & & & & & & Restricted \\
\hline & & & Porina atroperiostiola Makhija \& al. & Crustose & & + & & & & & & & & Restricted \\
\hline & & & Porina cf. subpungens Malme & Crustose & & + & & & & & & & & Restricted \\
\hline & & & Porina karnatakensis Makhija \& al. & Crustose & & + & & & & & & & & Restricted \\
\hline & & & Porina lucida R. Sant. & Crustose & & + & & & & & & & & Restricted \\
\hline & & & Porina masonhalei Makhija \& Chitale & Crustose & & + & & & & & & & & Restricted \\
\hline & & & Porina sp. & Crustose & & & & + & & & & & & Restricted \\
\hline & & & Porina sp. A & Crustose & & + & & & + & & & & + & \\
\hline \multirow[t]{2}{*}{ Porpidiaceae } & 2 & 2 & $\begin{array}{l}\text { Immersaria cf. olivacea Calatayud \& } \\
\text { Rambold }\end{array}$ & Crustose & & & & + & & & & & & Restricted \\
\hline & & & $\begin{array}{l}\text { Koerberiella wimmeriana (Körber) B. } \\
\text { Stein }\end{array}$ & Crustose & & & + & & & & & & & Restricted \\
\hline \multirow[t]{7}{*}{ Pyrenulaceae } & 2 & 7 & $\begin{array}{l}\text { Lithothelium obtectum (Müll. Arg.) } \\
\text { Aptroot }\end{array}$ & Crustose & & + & & & & & & & & Restricted \\
\hline & & & Pyrenula cf. mastophoriza (Nyl.) Zahlbr. & Crustose & & + & & & & & & & & Restricted \\
\hline & & & $\begin{array}{l}\text { Pyrenula depressa (Müll. Arg.) Makhija } \\
\text { \& Chitale }\end{array}$ & Crustose & & + & & & & & & & & Restricted \\
\hline & & & Pyrenula glabrescens Vain. & Crustose & & + & & & & & & & & Restricted \\
\hline & & & Pyrenula sp. B & Crustose & & + & & & & & & & & Restricted \\
\hline & & & Pyrenula sp. C & Crustose & & + & & & & & & & & Restricted \\
\hline & & & Pyrenula sp. D & Crustose & & + & & & & & & & & Restricted \\
\hline \multirow[t]{2}{*}{ Ramalinaceae } & 4 & 14 & Bacidia albicerata (Kremp.) Zahlbr. & Crustose & & + & & & & & & & & Restricted \\
\hline & & & Bacidia alutacea (Kremp.) Zahlbr. & Crustose & & & & + & + & & & & & \\
\hline
\end{tabular}




\begin{tabular}{|c|c|c|c|c|c|c|c|c|c|c|c|c|c|c|}
\hline \multirow[t]{2}{*}{ Families } & \multirow[t]{2}{*}{ Genera } & \multirow[t]{2}{*}{ Species } & \multirow[t]{2}{*}{ Names of Taxon } & \multirow[t]{2}{*}{ Growth forms } & \multicolumn{2}{|c|}{ HLF } & \multicolumn{7}{|c|}{ BM } & \multirow[t]{2}{*}{ Status } \\
\hline & & & & & 1 & 2 & 3 & 4 & 5 & 6 & 7 & 8 & 9 & \\
\hline & & & Bacidia fusconigrescens (Kremp.) Zahlbr. & Crustose & & + & & + & & & & & & \\
\hline & & & Bacidia incongruens (Stirt.) Zahlbr. & Crustose & & + & & & & & & & & Restricted \\
\hline & & & Bacidia personata Malme & Crustose & & & & + & & & & & & Restricted \\
\hline & & & Bacidia rubella (Hoffm.) A. Massal. & Crustose & + & & & + & & & & & & \\
\hline & & & Bacidia sp. A & Crustose & + & & & & & & & & & Restricted \\
\hline & & & Bacidia subacerina Nyl. ex Vain. & Crustose & & & & & + & & & & & Restricted \\
\hline & & & Bacidia submedialis (Nyl.) Zahlbr. & Crustose & & + & & & & & & & & Restricted \\
\hline & & & $\begin{array}{l}\text { Lopezaria isidiza (Makhija \& Nagarkar) } \\
\text { Aptroot \& Sipman }\end{array}$ & Crustose & & & & + & & & & & & Restricted \\
\hline & & & $\begin{array}{l}\text { Phyllopsora breviuscula (Nyl.) Müll. } \\
\text { Arg. }\end{array}$ & Squamulose & + & & & & & & & & & Restricted \\
\hline & & & $\begin{array}{l}\text { Phyllopsora corallina (Eschw.) Mull. } \\
\text { Arg. }\end{array}$ & Squamulose & & + & & + & & & & & & \\
\hline & & & Phyllopsora foliata (Stirt.) Zahlbr & Squamulose & + & + & & & & & & & & \\
\hline Rocellaceae & 1 & 1 & $\begin{array}{l}\text { Enterographa micrographa (Nyl.) } \\
\text { Redinger }\end{array}$ & Crustose & + & + & & & & & & & & \\
\hline \multirow[t]{3}{*}{ Stereocaulaceae } & 1 & 3 & Lepraria coriensis (Hue) Sipman & Leprose & & & & + & & & & & & Restricted \\
\hline & & & Lepraria lobificans Nyl. & Leprose & & & & + & & & & & & Restricted \\
\hline & & & Lepraria sp. & Leprose & & & & + & & & & & & Restricted \\
\hline \multirow[t]{8}{*}{ Teloschistaceae } & 1 & 8 & Caloplaca abuensis Joshi \& Upreti & Crustose & & & & + & & & & & & Restricted \\
\hline & & & Caloplaca amarkantakana Joshi \& Upreti & Crustose & & & & + & & & & & & Restricted \\
\hline & & & Caloplaca cupulifera (Vain.) Zahlbr. & Crustose & & & & + & & & & & & Restricted \\
\hline & & & $\begin{array}{l}\text { Caloplaca flavorubescens (Huds.) J.R. } \\
\text { Laundon }\end{array}$ & Crustose & & & & + & & & & + & & \\
\hline & & & $\begin{array}{l}\text { Caloplaca herbidella (Nyl. ex Hue) H. } \\
\text { Magn. }\end{array}$ & Crustose & & + & & & + & & & & & \\
\hline & & & Caloplaca inconspecta Arup & Crustose & & & & & + & & & + & & \\
\hline & & & Caloplaca pollinii (A. Massal.) Jatta & Crustose & & & & + & & & & & & Restricted \\
\hline & & & Caloplaca sp. A & Crustose & & & + & & & & & & & Restricted \\
\hline \multirow[t]{7}{*}{$\begin{array}{l}\text { Thelotremoid- } \\
\text { Graphidaceae }\end{array}$} & 5 & 14 & $\begin{array}{l}\text { Chapsa laceratula (Müll. Arg.) Rivas } \\
\text { Plata \& Lücking }\end{array}$ & Crustose & + & + & & & & & & & & \\
\hline & & & Chapsa leprocarpa (Nyl.) A. Frisch & Crustose & + & & & & & & & & & Restricted \\
\hline & & & Chapsa sp. A & Crustose & & + & & & + & & & & & \\
\hline & & & Chapsa sp. B & Crustose & & + & & & & & & & & Restricted \\
\hline & & & $\begin{array}{l}\text { Diploschistes } \quad \text { rampoddensis } \\
\text { Zahlbr. }\end{array}$ & Crustose & & & + & & & & & + & & \\
\hline & & & $\begin{array}{l}\text { Diploschistes cf. rampoddensis (Nyl.) } \\
\text { Zahlbr. }\end{array}$ & Crustose & & & & + & & & & & & Restricted \\
\hline & & & Diploschistes sp. A & Crustose & & + & & & & & & & & Restricted \\
\hline
\end{tabular}




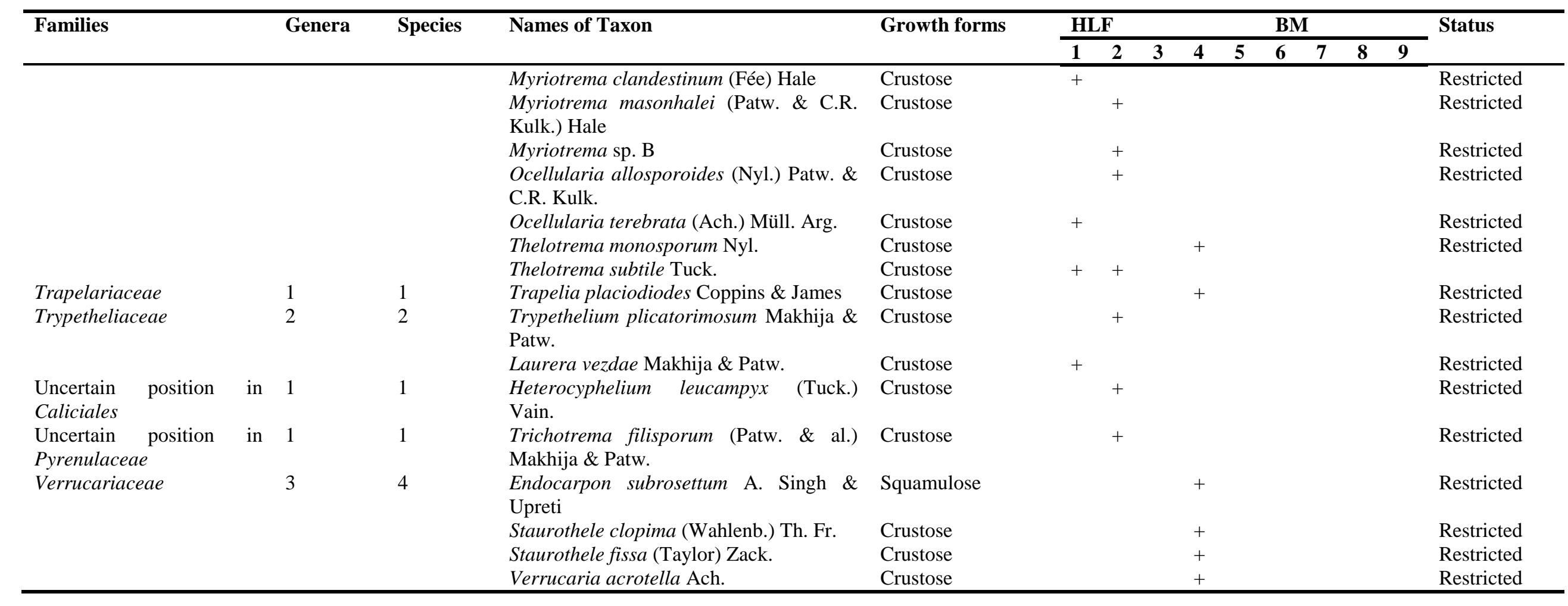


The total number of crustose forms is 151 spp, foliose 69 spp, fruticose six species, leprose three species squamulose six species and crustose-squamulose-foliose one species. Lichens were seen to utilize diverse substrates on the plateaus, such as rock, bark, soil and also sometimes mosses, 23 species are exclusively saxicolous (on rocks and boulders) which is $9.82 \%$ of the total plateau lichens, three species are seen share both bark and rock surfaces, 203 species are exclusively corticolous (on bark) which is $86.75 \%$, four species exclusively terricolous (on soil), three species exclusively muscicolous (on moss) and two species are seen share both the barks and moss. (TABLE 4) and the remaining species are all corticolous as per the list (TABLE 5). The majority of the species ca. 158 species are restricted to one plateau, whereas 24 species are common to more than four plateaus, while the remaining have an apparently rare status. Though these areas appear barren and rocky the barren part covers $9.82 \%$ of lichen cover of the total plateau area. Further systematic studies might give a better idea regarding regional distribution of species. However, it is clear that speciation on these terrestrial island habitat continues even now.

Similarly various inselbergs, duricrust, limestone, and quartzite rich, granite outcrops have been explored for their lichen studies worldwide and there is a mention that "Every single rock outcrop and quary supported at least one red-listed cryptogam species and among lichens the central European endemic Endocarpon latzelianum was found new to Germany" (Thiel \& Spribille 2007).

Thus detailed studies will enhance knowledge of lichen diversity on these poorly explored plateaus. The isolation or fragmentation of ecological habitats can have significant impact on biodiversity. Fragmentation may disrupt ecological processes critical to the maintenance of biodiversity, especially if over long periods. Hence rehabilitation of areas within short periods and maintaining ecological corridors become necessary mitigation measures. Introduction of burning as weed control can disrupt natural ecosystems. The recovery of lichens is very slow.

This list will be helpful at the national and regional levels to protect and raise government and public interest to help identify lichens to the specific national conservation action programs. Nevertheless, a number of lichens occurring on the basaltic rock at great heights, have never been studied due to difficulties in collection. Such studies will certainly result in many new and interesting lichens.

\section{Acknowledgements}

The author is grateful to the Department of Science and Technology-SERB, SB/FT/LS187/2012 New Delhi for the financial support; the Director, Agharkar Research Institute, Pune, for the laboratory facilities, I also thank the authorities of Maharashtra State Biodiversity Board and also the Forest Department for their permission in the surveys. Thanks are also due to Dr. Aparna Watve for reviewing the manuscript, also many thanks to Subhash Gaikwad \& Bharati Sharma for their technical assistance, timely help and suggestions. Last but not the least, I also thank the anonymous reviewers for their valuable suggestions.

\section{References}

Bhattaria U, Tetali P, Kelso S. 2012 - Contributions of vulnerable hydrogeomorphic habitats to endemic plant diversity on the Kas plateau, Western ghats. Springer Plus 1, 25, http: /dx.doi.org/10.1186/2193-1801-1-25.

Büdel B. 1999 - Ecology and diversity of rock-inhabiting cyanobacteria in tropical regions. European Journal of Phycology 34(4), 361-370.

Giri VB, Gower DJ, Wilkinson M. 2004 - A new species of Indotyphlus Taylor (Amphibia: Gymnophiona: Caeciliidae) from the Western Ghats, India. Zootaxa 739, 1-19.

Giri VB, Bauer AM. 2008 - A new ground-dwelling Hemidactylus (Squamata: Gekkonidae) from Maharashtra, with a key to the Hemidactylus of India. Zootaxa 1700, 21-34.

Gower DJ, Giri VB, Wilkinson M. 2007 - Rediscovery of Gegeneophis seshachari Ravichanddran, Gower \& Wilkinson, 2003 Amphibia: Gymnophiona: Caeciliidae) at the type locality. Herpetozoa, 19(3/4), 121-127. 
Lekhak, MM, Yadav SR. 2012 - Herbaceous vegetation of threatened high altitude lateritic plateau ecosystems of Western Ghats, southwestern Maharashtra, India. Rheedea, 22(1), 39-61.

Makhija U, Chitale G, Dube A. 2014 - Lichens of Maharashtra. Published by Gajendra Singh Gahlot for Bishen Singh Mahendra Pal Singh, Dehra dun, India. ISBN : 978-81-211-0865-2.

Pandit GS. 2014b - Lichens of the Mahabaleshwar Panchgani Ecosensitive zone (MPESZ), Maharashtra, India. Journal of Threatened Taxa 6(5), 5784-5791; http://dx.doi.org/10.11609/JoTT.o3784.5784-91.

Pandit GS. 2014a - Immersaria and Koerberiella, two new generic records to India. Current Research in Environmental \& Applied Mycology 4(1), 137-140, Doi 10.5943/cream/4/1/12.

Porembski S, Becker U, Seine R. 2000 - Islands on islands: habitats on Inselbergs. Ecological Studies 146: 49-67. In: Porembski, S. \&W. Barthlott (eds.). Inselbergs - Biotic Diversity of Isolated Rock Outcrops in Tropical and Temperate Regions. Ecological studies Springer, 146, Heidelberg.

Singh KP, Sinha GP. 2010 - Indian Lichens an Annotated checklist. Publ. by Government of India. Botanical Survey of India. Shiv Offset Press Dehra Dun,; ISBN 978-81-8177-036-3.

Thiel H, Spribille T. 2007 - Lichens and Bryophytes on shaded sandstone outcrops used for rock climbing in the vicinity of Göttinggen (southern Lower Saxony, Germany). Herzogia 20, 159177.

Watve A. 2013 - Status review of Rocky plateaus in the northern Western Ghats and Konkan region of Maharashtra, India with recommendations for conservation and management. Journal of Threatened Taxa 5(5), 3935-3962.

Widdowson M, Cox KG. 1996 - Uplift and erosional history of the deccan traps India: Evidence from laterites and drainage patterns of the Western Ghats and Konkan coast. Earth and Planetary Science Letters 137, 57-69.

Yadav SR, Sardesai MM. 2002 - Flora of Kolhapur District. Shivaji University, Kolhapur, 1-679 pp. 